\title{
Supplier BIM competence assessments within the cloud: a proposed Fuzzy-TOPSIS approach
}

\author{
A.-M. Mahamadu, L. Mahdjoubi \& C. A. Booth \\ Construction and Property Research Centre, \\ University of the West of England (UWE), UK
}

\begin{abstract}
Building information modelling (BIM) is regarded as a technological solution for construction supply chain (SC) integration through centralised digital communications. A number of approaches have been proposed for the evaluation of a firm's capability to deliver BIM. However, none of the existing approaches have been specifically tailored for supplier selection processes. Most of the existing tools have been developed for individual firm capability or maturity evaluations rather than cross comparative assessments for the purpose of selection. Consequently, there remains a lack of tools for prioritising suppliers based on their ability to deliver BIM during evaluations for selection. A Fuzzy-TOPSIS computational framework for implementing such cross comparative assessments is proposed. This method is proposed in view of its suitability for implementation within a cloud-based decision support environment. A high-level architecture for a cloud-based decision support tool (DST) which can incorporate the proposed computational model is presented. The proposed framework is capable of enhancing decision making during the selection process through a robust approach to the aggregation and comparison of SC firm's BIM competence and readiness. Keywords: BIM, supply chain, selection, cloud, decision support.
\end{abstract}

\section{Introduction}

Building information modelling (BIM) describes an embodiment of policies, processes and technologies for the generation and management of project data in digital formats throughout a facility's life-cycle [1]. BIM is expected to bridge communication gaps which have led to a lack of collaboration and integration 
within the construction supply chain (SC) [2]. Organisations are therefore developing the necessary capabilities to enable them deliver projects through BIM [3]. Evidence, however, points to inconsistency in the levels of adoption due to varying degrees of proficiency across the construction SC [4]. This is exacerbated by a lack of standardised approach for evaluating a supplier's ability to deliver BIM especially during selection or prequalification process.

\section{BIM competence and supplier selection}

Suppliers in the construction SC context refers to consultants, subcontractors and construction service providers who are employed for the purposes of completing projects [2]. Suppliers are selected either as part of a project or a construction team for the execution of several projects. In order to be selected, suppliers normally undergo a qualification process where they are appraised to ascertain their suitability or competence. The selection process is one of the most important phases of a project in view of its usefulness in reducing the risk of engaging incapable suppliers [5]. As a result, there is a need for robust methodologies for prioritising potential candidates based on attributes that show necessary capability. Recently, an important area in which suppliers need to show capability is BIM [3, 6]. However, despite the proliferation of capability assessment frameworks, there remains a lack of suitable tools for assessing SC firm's ability to deliver BIM during the selection phase.

\section{BIM competence and readiness assessments}

The general lack of appropriate techniques and tools for evaluating BIM capability has resulted in the 'proliferation of BIM wash' [7]. According to Succar [7] this refers to false claims about ability to deliver in BIM. Main contractors and clients cannot, therefore, appropriately assess such claims as a result of the lack of benchmarks in the determination of competence, proficiency and willingness to deliver through BIM $[6,7]$. Existing frameworks have been developed mainly to assess firm capability and maturity, arguable making the more suitable for internal implementation evaluations [3, 4, 6, 7]. Others were developed for project level performance measurement thus making them suitable for internal performance measurement purposes [8]. The tools with some relevance for the selection activities were, however, not developed for the SC context neither do their methodologies of evaluation allow for direct cross comparison of alternative firms $[4,6]$. They require the appraisal of individual firms in order to allocate an aggregated score for their performance. However, the selection process generally requires a more robust approach to comparing firms' performance across multiple assessment criteria [5,9]. This has led to the advocacy for the use of multi-criteria decisions support techniques to aid more robust mathematical modelling of selection decisions $[5,9,10]$. Existing frameworks for assessing BIM performance mostly rely on rather simplistic addition of weighted scores which are less robust for evaluating several alternatives across multiple criteria especially for the purposes of prioritisation or ranking. 


\section{Computational methods for construction firms and supplier selection}

Recently there has been greater emphasis on the use of multi-criteria decision support methodologies for the selection of contractors, sub-contractors and consultants. Several computational techniques have been relied on in the development of prequalification and selection models or tools. They include the dimensional weighting model [10]; multi-attribute analysis and utility theory [5]; case-based reasoning system for the capture and reuse experimental knowledge experts in evaluation models [11]; neural networks (NN) for matching contractors' attributes to the clients objectives [12]; Analytical Hierarchy Process (AHP) or its fuzzy extension [13, 14]; ANP and Monte Carlo simulations [15]; and fuzzy set theory $[9,16]$. These models however predate BIM, thus, do not include criteria related to BIM. However, criteria used in other BIM performance and capability frameworks, provide some basis for adaptation into specifically developed selection models for BIM. Some of the relevant criteria cited include: organisations and staff competence; culture; availability of technical, managerial and administrative resources [3, 4, 6, 7, 17]. According to Succar et al. [17], BIM assessment criteria should be any attributes that show a firm's BIM competence, available resources and historical indicators of BIM performance.

\section{Proposed methodology}

Despite the availability of many multi-criteria decision methods (MCDM), there are many limitations which render them unsuitable for the selection of BIM competent suppliers. Some approaches are restrictive due to complexity, thus, require a good knowledge of mathematics in order to apply them $[5,9,10]$. Similarly, most generally fail to recognise the uncertainty and vagueness associated with evaluating several alternatives by human decision makers [16]. Hence, there is a need for the proposition of models that are capable of alleviating these limitations as well as capable of being applied within a web or cloud based environment for the development of DSTs. In this paper, Fuzzy-TOPSIS framework is proposed for ranking alternative suppliers on the basis of their BIM competence or readiness.

\subsection{Proposed computational method for prioritising alternatives suppliers}

The proposed Fuzzy-TOPSIS model for prioritisation of BIM competent suppliers will require decision makers to decide on the requisite criteria for a particular evaluation under consideration. These criteria must meet the BIM project objectives or client requirements. The relative importance of such criteria should also be determined through allocation of criteria weights. The Delphi method is proposed for the determination of criteria and criteria importance (weight) in this study. The Delphi method is an iterative process used to collect and analyze variations in the judgments of experts using a series of questionnaires interspersed 
with feedback $[4,18]$. The next step is the prioritisation of alternative suppliers based on the agreed criteria. This process is depicted in Figure 1 below.

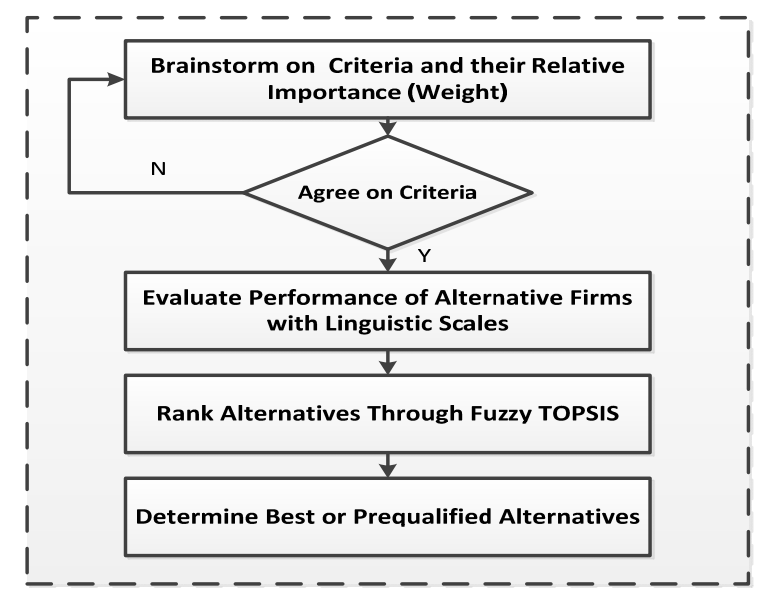

Figure 1: Generic steps for implementing Fuzzy-TOPSIS method in prioritising BIM competent suppliers.

\subsubsection{Fuzzy-Technique for Order of Preference by Similarity to Ideal Solution (TOPSIS)}

An MCDM technique is deemed appropriate for ranking alternative suppliers based on BIM competence, hence the choice of Fuzzy-TOPSIS. Hwang and Yoon [19] developed TOPSIS (Technique for Order Performance by Similarity to Idea Solution) for determination of the best alternative through the measurement of the Euclidean distance from best scenario. TOPSIS is based on the concept that the chosen alternative should have the shortest geometric distance from the positive ideal solution and the longest geometric distance from the negative ideal solution $[19,20]$. Furthermore, decision makers often find it challenging to allocate crisp figures for different types of criteria especially when they are subjective or complicated to measure [20]. Interval judgments however make allocation of preference scores very easy especially when used for comparative judgements. In order to perform a TOPSIS evaluation, universal scales are normally used to make attributes comparable. This is achieved through the use of common linguistic scales for assessing each criteria. For example software capability can be rated on scales with corresponding linguistic variable between Very Low to Very High. However, a critical limitation of the classical TOPSIS is the inability to cater for uncertainty and imprecision associated with human decision making [21]. Fuzzy set theory has been integrated with TOPSIS to mathematically model and reduce inconsistencies that can be introduced by subjectivity [22]. Zadeh's [23] fuzzy set theory, allows a continued assessment of the membership of elements contained in a set. The membership is defined as the function representing the real unit interval $[0,1][23]$. The incorporation of fuzzy set principles into TOPSIS allows the modelling of vagueness as well as incomplete information [20]. This 
eliminates the challenges relating to imprecision and vagueness when judgement is based only on crisp values [21]. In this framework, the proposed model uses triangular fuzzy number for the Fuzzy-TOPSIS computations. Triangular fuzzy approach is simple to use as well as easy to understand [20]. Triangular fuzzy have been extensively applied when fuzzy is integrated with decision modelling techniques. The basic definitions and steps for the triangular fuzzy as defined by Dağdevirena et al. [20] and Wang and Chang [24] is presented below.

The fuzzy set $\tilde{A}$ in the universe of discourse $X$ is part of a membership function $\mu_{\tilde{A}}(x)$ and is associated with each element $x$ in $X$, between a real number interval $[0,1]$. The function $\mu_{\tilde{A}}(x)$ is the grade of membership of $x$ in the fuzzy set. A triangular fuzzy number $\tilde{a}$ is defined as a triplet $\left(a_{1}, a_{2}, a_{3}\right)$, Figure 2. The membership function $\mu_{\tilde{a}}(x)$ is defined as (Equation (1)):

$$
\mu_{\tilde{a}}(x)=\left\{\begin{array}{cc}
0, & x<a_{1} \\
\frac{x-a_{1}}{a_{2}-a_{1}}, & a_{1} \leq x \leq a_{2} \\
\frac{x-a_{3}}{a_{2}-a_{3}}, & a_{2} \leq x \leq a_{3} \\
0, & x>a_{3}
\end{array}\right.
$$

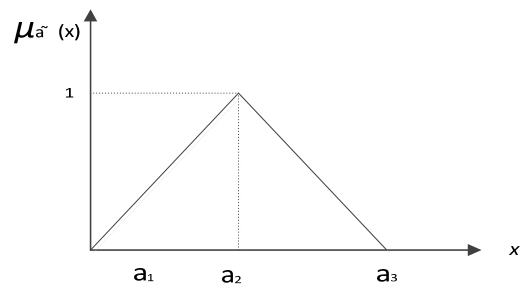

Figure 2: $\quad$ Triangular fuzzy number $\tilde{a}$.

Let $\tilde{a}$ and $\tilde{b}$ be two triangular fuzzy numbers, parameterized by the triplet $\left(a_{1}\right.$, $\left.a_{2}, a_{3}\right)$ and $\left(b_{1}, b_{2}, b_{3}\right)$ with the following (Equations (2)-(6)):

$$
\begin{gathered}
\tilde{a}(+) \tilde{b}=\left(a_{1}, a_{2}, a_{3}\right)(+)\left(b_{1}, b_{2}, b_{3}\right)=\left(a_{1}+b_{1}, a_{2}+b_{2}, a_{3}+b_{3}\right), \\
\tilde{a}(-) \tilde{b}=\left(a_{1}, a_{2}, a_{3}\right)(-)\left(b_{1}, b_{2}, b_{3}\right)=\left(a_{1}-b_{1}, a_{2}-b_{2}, a_{3}-b_{3}\right), \\
\tilde{a}(\times) \tilde{b}=\left(a+1, a_{2}, a_{3}\right)(\times)\left(b_{1}, b_{2}, b_{3}\right)=\left(a_{1}, b_{1}, a_{2} . b_{2}, a_{3} . b_{3}\right), \\
\tilde{a}(/) \tilde{b}=\left(a_{1}, a_{2}, a_{3}\right)(/)\left(b_{1}, b_{2}, b_{3}\right)=\left(a_{1} / b_{3}, a_{2} / b_{2}, a_{3} / b_{1}\right), \\
\tilde{a}=\left(k a_{1}, k a_{2}, k a_{3}\right) .
\end{gathered}
$$

BIM competence criteria would normally include both objective and subjective items $[3,4,6,17]$. Where the evaluation team relies on many subjective criteria, assessments will generally be based on approximations, making crisp value allocation challenging. In such a case, linguistic judgement scales are preferable. 
Objectives criteria can however remain crisp especially if an acceptable method of allocating values to each alternative exists. The linguistic variables used can then be represented by corresponding fuzzy numbers as presented in Figure 3 and Table 1.

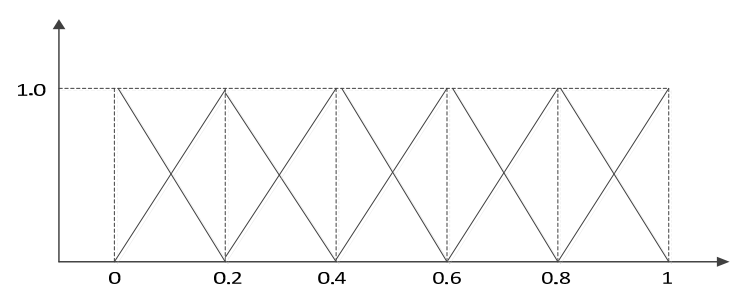

Figure 3: Membership function of linguistic values for criteria rating.

Table 1: Linguistic values for subjective judgements.

\begin{tabular}{|c|l|c|c|}
\hline Level & \multicolumn{2}{|l|}{ Example } & Fuzzy Number \\
\hline V1 & Very Poor & VP & $(0,0,0.2)$ \\
\hline V2 & Poor & P & $(0,0.2,0.4)$ \\
\hline V3 & Average & A & $(0.2,0.4,0.6)$ \\
\hline V4 & Good & G & $(0.4,0.6,0.8)$ \\
\hline V5 & Very Good & VG & $(0.6,0.8,1)$ \\
\hline V6 & Outstanding & O & $(0.8,1,1)$ \\
\hline
\end{tabular}

Let $\tilde{a}=\left(a_{1}, a_{2}, a_{3}\right)$ and $\tilde{b}=\left(b_{1}, b_{2}, b_{3}\right)$ be two triangular fuzzy numbers. The vertex method for computing the distance between them can be expressed as follows:

$$
\begin{aligned}
d(\tilde{a}, \tilde{b}) & =\sqrt{\frac{1}{3}\left[\left(a_{1}-b_{1}\right)^{2}+\left(a_{2}+b_{2}\right)^{2}+\left(a_{3}-b_{3}\right)^{2}\right]} \\
\tilde{V} & =\left[\tilde{v}_{i j}\right]_{n \times j} \quad i=1,2, \ldots . n, j=1,2, \ldots ., j,
\end{aligned}
$$

where:

- $\tilde{v}_{i j}=\tilde{x}_{i j} \times w_{i}$

- A set of performance ratings of $A_{j}(j=1,2, \ldots . j)$ with respect to criteria $c_{i}(i=1,2, \ldots . n)$ called $\tilde{X}=\left\{\tilde{x}_{i j}, i=1.2 \ldots ., n, J=1,2, \ldots . . J\right\}$

- $\quad$ A set of importance criteria $w_{i}(i=1,2, \ldots . n)$.

Based on the above definitions the steps for Fuzzy-TOPSIS can be operationalised as follows:

Step 1: Select a linguistic value $\left(\tilde{x}_{i j}, i=1,2, \ldots ., n, J=1,2, \ldots . J\right)$ with respect to an evaluation of performance of an alternative for a particular criteria; 
Step 2: Calculate the weighted normalized fuzzy decision matrix;

Step 3: Determine the positive-ideal $\left(A^{*}\right)$ and negative ideal $\left(A^{-}\right)$solution. The fuzzy negative-ideal solution $\left(F N I S, A^{*}\right)$ and the fuzzy negative-ideal solution $\left(F N I S, A^{-}\right)$(Equations (9) and (10)).

$$
\begin{aligned}
& A^{*}=\left\{\tilde{v}_{1}^{*}, \tilde{v}_{2}^{*}, \ldots \ldots \ldots \tilde{v}_{i}^{*}\right\}=\left\{\left(\begin{array}{cc}
\max & \\
j & v_{i j} \mid i \in I^{\prime}
\end{array}\right) \times\left(\begin{array}{cc}
\min & \\
j & v_{i j} \mid i \in I^{\prime \prime}
\end{array}\right)\right\}, \\
& i=1,2, \ldots, n j=1,2, \ldots J \text {, } \\
& A^{*}=\left\{\tilde{v}_{1}^{-}, \tilde{v}_{2}^{-}, \ldots \ldots \ldots \tilde{v}_{i}^{-}\right\}=\left\{\left(\begin{array}{c}
\max \\
j
\end{array} v_{i j} \mid i \in I^{\prime}\right) \times\left(\begin{array}{c}
\min \\
j
\end{array} v_{i j} \mid i \in I^{\prime \prime}\right)\right\}, \\
& i=1,2, \ldots, n j=1,2, \ldots J \text {, }
\end{aligned}
$$

where $I^{\prime}$ is benefit criteria and $I^{\prime \prime}$ is cost criteria.

Step 4: Calculate the distance of each alternative from $A^{*}$ and $A^{-}$(Equations (11) and (12)):

$$
\begin{aligned}
& D_{j}^{*}=\sum_{j=1}^{n} d\left(\tilde{v}_{i j}, \tilde{v}_{i}^{*}\right) j=1,2, \ldots, J \\
& D_{j}^{-}=\sum_{j=1}^{n} d\left(\tilde{v}_{i j}, \tilde{v}_{i}^{-}\right) j=1,2, \ldots, J
\end{aligned}
$$

Step 5: Calculate similarities to ideal solution (Equation (13)):

$$
C C_{j}=\frac{D_{j}^{-}}{D_{j}^{*}+D_{j}^{-}} j=1,2, \ldots J .
$$

Step 6: Rank alternatives based on their distance from ideal situation.

In order to implement this model, a tender or decision making team must assess each firm in relation to each criteria (weighted) using the appropriate linguistic variable on the scales provided. Figure 4 shows the generic decision hierarchy for implementing the Fuzzy-TOPSIS model. This computational model can be implemented as part of a wider computational framework a cloud-based decision support tool (DST).

\section{Implementation of proposed computational model in cloud-based decision support environment}

The construction industry is looking towards the optimization of IT in all operations [2]. One of the most advocated platforms being the internet. Cloud computing has been advocated as a useful medium for implementing construction applications and tools $[25,26]$. Cloud computing refers to a variety of web or internet based concepts and methods for sharing configurable computing resources (e.g. networks, servers, storage, applications, and services) [25]. Cloud computing provides cheap or free access to many computational services offered in the 


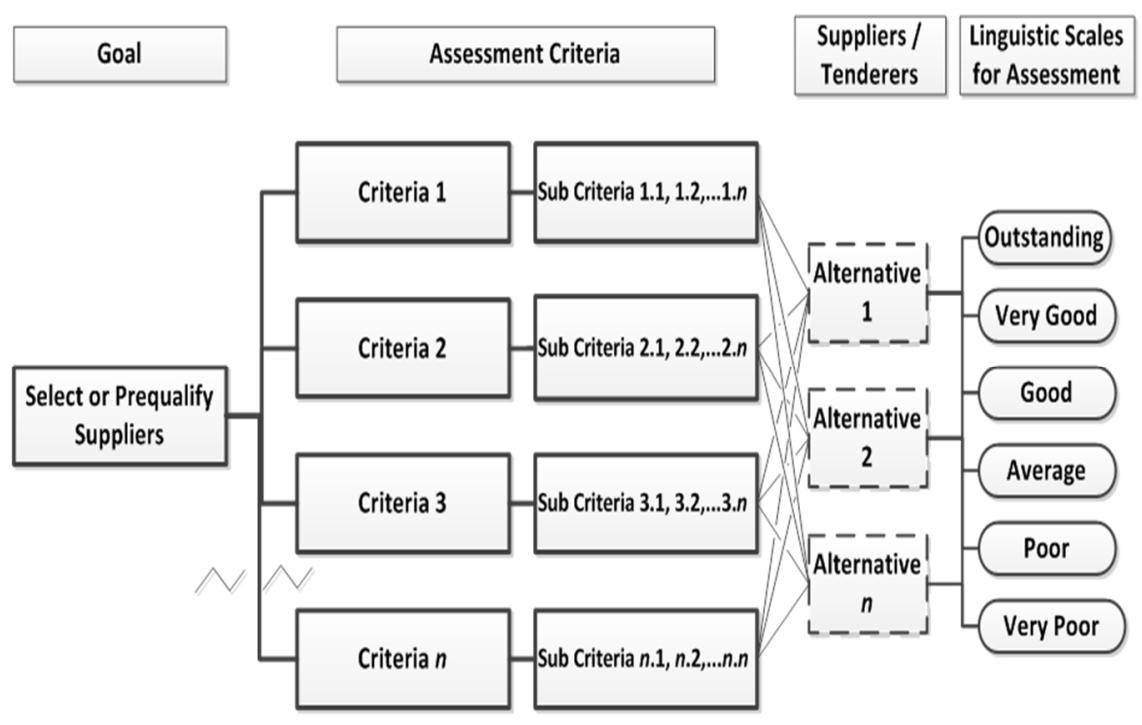

Figure 4: Sample decision hierarchy for implementing Fuzzy-TOPSIS model.

internet as a service [26]. A DST implemented within the web refers to applications or software that aids the integration of multiplicity of data sources and tools for the generation of relevant information needed for taking a decision [27]. The benefits of such systems include implantation within the cloud, low cost of operations as a lot or free applications exist both for their design and use. Moreover, cloud-based applications do not require additional software to operate as they are often accessible through most user interfaces such as browsers from computers or mobile devices [25]. Despite concerns about security, several approaches exist for safeguarding data through design of associated systems and processes [26].

\subsection{Proposed cloud-based decision support architecture}

The architectural components of the proposed cloud-based DST platform include the web interface, a presentation layer as well as cloud services and data layer for hosting database and computational models including the proposed FuzzyTOPSIS framework. Data required for the Fuzzy-TOPSIS computation will be supplied by evaluators through a user interface as well as stored data in the DST's databases. The schematic diagram showing the basic high-level architecture is presented in Figure 5.

JavaScript is proposed for client side scripting as it is one of the most widely used in web browsers. It is also appropriate where client-side scripts require interaction with the user or asynchronous communication and control [28]. According to Flanagan and Ferguson [28], JavaScript is also a multi-paradigm language for supporting object-oriented programming. HTML (Hyper Text 


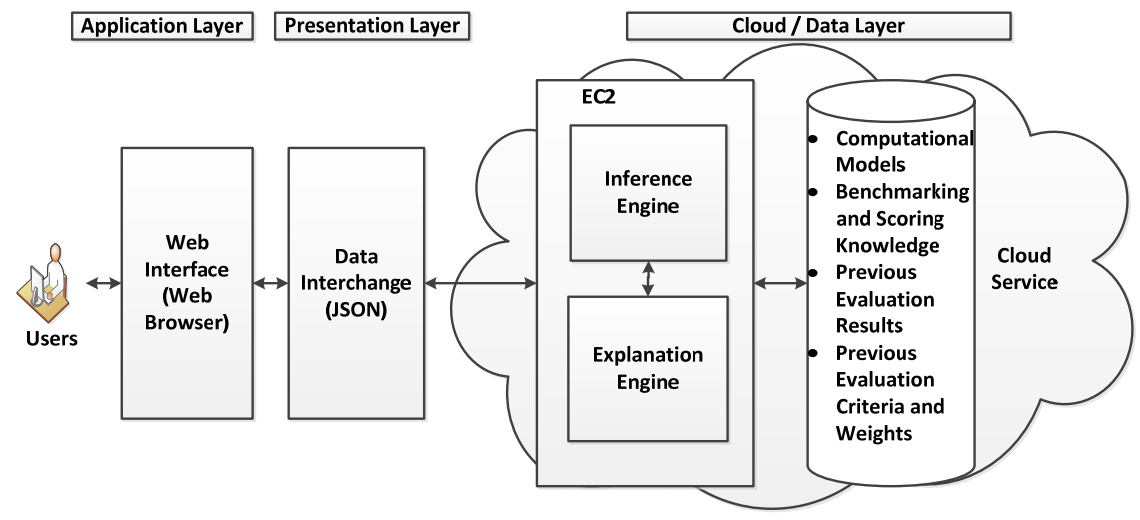

Figure 5: High-level architecture for cloud-based DST.

Markup Language) is proposed as mark-up language. Most browsers use HTML which describes the structure of a website semantically along with cues for presentation [29]. According to Arslan et al. [29], CSS (Cascading Style Sheets) open standards, can aid to specify a layout or formatting that proper ties the HTML elements as well as provide dynamic graphical user interface (GUI). Proposed server side scripting language is PHP (Hypertext Pre-processor). PHP is a commonly used code and can be mixed with HTML codes and various templating engines and web frameworks [30]. JSON (JavaScript Object Notation) is also proposed for data representation. JSON is an open standard format and uses human-readable text to transmit data objects [31]. JSON aids transmission of data between server and web application. The http (Hypertext Transfer Protocol) transfer protocol between will support transfer of information between user interface and application [29].

A free cloud service is proposed for implementing the computational and database models through virtual resources. An example of such service is the Amazon Elastic Compute Cloud (EC2) [32]. This can support the operation of main functions of the DST especially in relation to the receipt of queries and processing within the inference and explanation engine components. The inference and explanation engine in decision support systems are used for storage and execution of the logical rules for computational analysis and retrieval of data [33]. A cloud-based dynamic database service is proposed to house the various knowledge and data including the Fuzzy-TOPSIS computational model, previous assessment results, criteria and criteria weights from historic assessments. Filters that are proposed for partitioning knowledge or data in the dynamic database are: the type and size of supplier; project type; project complexity; scale of project; BIM maturity and BIM complexity. This will ensure the storage and reuse of knowledge for projects with matching or similar attributes. 


\section{Conclusion}

The proposed system described, provides a robust approach for achieving a computational model for prioritising and ranking SC firms based on their BIM competence or readiness. This computational model can be incorporated within a broader framework for implementing a DST in a cloud computing environment. This framework is proposed to aid the development of a DST, specifically tailored for the selection phase of projects or for the purposes of SC management by main contractors and clients. The proposed DST framework allows users to register and enter project attributes which will include assessment criteria and details of alternative suppliers to be evaluated. Users will be required to provide weight for the criteria based on their relative importance in the evaluation scenario. Finally, users will perform evaluation of alternatives with the aid of linguistic scales from the Fuzzy-TOPSIS computational model. Results will then be displayed as well as stored as knowledge to aid future evaluations. The proposed framework, thus, provides a dynamic and cost effective approach for decision making during the selection of suppliers to work on BIM projects and environments.

\section{References}

[1] Eastman, C., Teicholz, P., Sacks, R. \& Liston, K. BIM handbook: A guide to Building Information Modeling, for Owners, Managers, Designers, Engineers, and Contractors, John Wiley and Sons: Hoboken, 2008.

[2] Vrijhoef, R. Supply chain integration in the building industry: The emergence of integrated and repetitive strategies in a fragmented and project-driven industry, IOS Press: Amsterdam, 2011.

[3] Succar, B., Sher, W. \& Williams, A. Measuring BIM Performance: Five Metrics. Architectural Engineering and Design Management, (2), pp. 120$142,2012$.

[4] Giel, B. \& Issa, R. Framework for Evaluating the BIM Competencies of Building Owners. Computing in Civil and Building Engineering, pp. 552559, 2014.

[5] Holt, G.D., Olomolaiye, P.O. \& Harris, F.D. Evaluating Pre-qualification Criteria in Contractor Selection, Building and Environment, 29 (4), 437448, 1994.

[6] Van Berlo, L., Dikkmans, T., Hendriks, H., Spekkink, D. \& Pel, W. BIM QuickScan: Benchmark of Performance in the Netherlands, Proceedings 29th International Conference, CIB W78 2012, October 17-19. Beirut, Lebanon, 2012.

[7] Succar, B. The five components of BIM Performance Measurement. CIB: Salford, 2010.

[8] Kam, C., Senaratna, D., Xiao, Y. \& McKinney, B. The VDC Scorecard. Center for Integrated Facility Engineering, Stanford University, 2013. Online http://vdcscorecard. stanford.edu 
[9] Nieto-Morote, A. \& Ruz-Vila, F. A fuzzy multi-criteria decision making model for construction contractor prequalification. Automation in Construction, 25, pp. 8-19, 2012.

[10] Jaselskis, E.J. \& Russell, J.S. An efficiently structured approach for selection of most promising construction contractors. Project Management Journal, 12(4), pp. 31-39, 1991.

[11] Ng, S.T. EQUAL: a case-based contractor prequalifier. Automation in Construction, 10, pp. 443-457, 2001.

[12] Lam, K.C., Hu, T., Ng, S.T., Skitmore, M. \& Cheung, S.O. A fuzzy neural network approach for contractor prequalification. Construction Management and Economics, 19, pp. 175-180, 2001.

[13] Fong, S.P. \& Choi K.S. Final Contractor Selection Using the Analytical Hierarchy Process. Construction Management and Economics, 18, pp. 547$557,2000$.

[14] Hosny, O., Nassar, K. \& Esmail, Y. Prequalification of Egyptian construction contractors using fuzzy-AHP models. Engineering, Construction and Architectural Management, 20(4), pp. 381-405, 2013.

[15] El-Abbasy, M.S., Zayed, T., Ahmed, M., Alzraiee, H. \& Abouhamad, M. Contractor Selection Model for Highway Projects Using Integrated Simulation and Analytic Network Process. Journal of Construction Engineering and Management, 139(7), pp. 755-767, 2013.

[16] Plebankiewicz, E. A fuzzy sets based contractor prequalification procedure. Automation in Construction, 22, pp. 433-443, 2012.

[17] Succar, B., Sher, W. \& Williams, A. An integrated approach to BIM competency acquisition, assessment and application. Automation in Construction, 35, pp. 174-189, 2013.

[18] Rowe, G. \& Wright, G. The Delphi Technique as a Forecasting Tool: Issues and Analysis. International Journal of Forecasting, 15, pp. 353-375, 1999.

[19] Hwang, C.L. \& Yoon, K. Multiple attribute decision making: Methods and applications, A State of the Art Survey. Springer-Verlag: New York, 1981.

[20] Dağdevirena, M., Yavuzb, S. \& Kılınçc, N. Weapon Selection using the AHP and TOPSIS Methods Under Fuzzy Environment. Expert Systems with Applications, 36(4), pp. 8143-8151, 2009.

[21] Tan, Y., Shen, L., Langston, C. \& Liu, Y. Construction project selection using Fuzzy-TOPSIS approach. Journal of Modelling in Management, 5(3), pp. 302-315, 2010.

[22] Wang, Y.M. \& Elhag, T.M.S. Fuzzy-TOPSIS method based on alpha level sets with an application to bridge risk assessment. Expert Systems with Applications, 31, pp. 309-319, 2006.

[23] Zadeh, L. A. Fuzzy sets. Information and Control, 8, pp. 338-353, 1965.

[24] Wang, T. C. \& Chang, T. H. Application of TOPSIS in evaluating initial training aircraft under a fuzzy environment. Expert Systems with Applications, 33, pp. 870-880, 2007.

[25] Redmond, A., Hore, A., Alshawi, A. \& West, R. Exploring how information exchanges can be enhanced through Cloud BIM. Automation in Construction, 24, pp. 175-218, 2012. 
[26] Mahamadu, A.M., Mahdjoubi, L. \& Booth, C. (2013). Challenges to BIMcloud integration: Implication of security issues on secure collaboration. Proceedings of the 5th IEEE International Conference on Cloud Computing Technology and Science, pp. 209-214, 2013.

[27] Mohemad, R., Hamdan, A.R., Ali, Othman, Z. \& Noor, N.M. Decision Support Systems (DSS) in Construction Tendering Processes. 7(2), pp. 3545, 2010.

[28] Flanagan, D. \& Ferguson, P. JavaScript: The Definitive Guide, 5th ed, O’Reilly \& Associates, 2006.

[29] Arslan, G., Kivrak, S., Birgonul, M. \& Dikmen, I. Improving Sub-contractor Selection Process in Construction Projects: Web-based Sub-contractor Evaluation System (WEBSES). Automation in Construction, 17, pp. 480488, 2008.

[30] Ide, A. PHP just grows and grows. 2013, Online. http://news.netcraft.com/archives/2013/01/31/php-just-grows-grows.html

[31] Bray, T. JSON Redux AKA RFC7159, 2014. Online. https://www.tbray.org/ongoing/When/201x/2014/03/05/RFC7159-JSON

[32] Amazon EC2, Online. http://aws.amazon.com/ec2/

[33] Hayes-Roth, F, Waterman, D. \& Lenat, D. Building Expert Systems. Addison-Wesley, 1983. 